\title{
EFFECT OF ENVIRONMENTAL FACTORS ON THE REPAIR OF BONE IN VITRO
}

\author{
G. C. Prasad, Varanasi, India, and J. J. Reynolds, Cambridge, England \\ From the Strangeways Research Laboratory, Cambridge
}

The dynamics of bone growth and repair have been extensively studied in vivo. The process of healing following the fracture of a long bone has been described by many workers, including McLean and Urist (1955), Ham and Leeson (1961) and Udupa and Prasad (1963). The use of organ culture for the study of bone growth, as originally described by Fell (1928), provided a new approach to the problem, but few studies of bone repair in vitro have been reported. Niven (1931), Bucher (1952) and Sevastikoglou (1962) described experiments on fractures in vitro and the healing of partial fractures has been examined by Shaw and Bassett (1964); in all this work complex natural media were used, but these are not suitable for the biochemical study of the direct action of vitamins, hormones and other agents on the process of repair. The development of a suitable chemically defined medium (Biggers, Gwatkin and Heyner 1961; Reynolds 1966) for the cultivation of skeletal elements, suggested that it might now be possible to elaborate a technique whereby a reproducible pattern of bone repair in vitro could be obtained in rigidly controlled conditions. In the experiments described in this paper, the histological and biochemical changes that take place in fractured embryonic chick tibiae, grown in a protein-free chemically defined medium, have been investigated. The results have shown that complete repair can be achieved by this method, which can be utilised for biochemical study.

\section{MATERIALS AND METHODS}

Explants and culture technique-The tibiae of fourteen-day chick embryos were used; at this age the tibia consists of a calcified shaft of bone containing haemopoietic tissue, and large cartilaginous ends. The bones, with intact periosteum, were isolated from the adherent tissues under a dissecting microscope and were kept immersed in Tyrode's solution during the dissection. The fractures were made by cutting completely through the middle of the shaft with a cataract knife. The two cut ends were then placed in close contact with each other on a shallow stainless steel grid. The culture chambers were similar to those described by Fell and Weiss (1965); they were incubated at 38.5 degrees Centigrade for up to sixteen days in a gas phase of either 75 per cent of nitrogen to 20 per cent of oxygen with 5 per cent of carbon dioxide, or 45 per cent of nitrogen to 50 per cent of oxygen with 5 per cent of carbon dioxide. In a later series of experiments the bony shafts were excised from the cartilaginous ends and then fractured in the usual way; the two pieces of shaft were then placed in apposition and grown in a similar manner to that described above for the fractured whole bones.

Culture medium-The chemically defined medium was prepared as described by Reynolds (1966) with the addition of ascorbic acid at a concentration of 150 micrograms per millilitre of medium. The medium was changed every second day. In some experiments insulin was added at a concentration of 500 micro-units per millilitre. Crystalline insulin (24.5 units per milligram) was purchased from Messrs Allen and Hanbury, London, and a concentrated solution was prepared in $0.001 \mathrm{~N}$ hydrochloric acid. This was then diluted into the medium. Measurement of length and weight-The lengths of the explants were measured every second day by means of a calibrated eye-piece grid. The wet weights were determined by blotting the bones on filter paper and weighing them in stoppered tubes. The explants (except those for histology) were then dried for forty-eight hours in vacuo over phosphorus pentoxide, and reweighed. 
Biochemical analyses-The hydroxyproline contents of the explants were determined by the method of Prockop and Udenfriend (1960), and the collagen content was obtained by multiplying the hydroxyproline content by $7 \cdot 46$. The amount of hexosamine in the explants was determined by the method of Boas (1953). In some experiments the used culture medium was analysed for glucose by an enzymatic method (Slein 1963).

Histology-Two fractured bones from each treatment were fixed on the fourth, eighth, twelfth and sixteenth day of culture, in acetic Zenker's solution, decalcified with formol-nitric acid and embedded in paraffin wax. Serial sections were cut at a thickness of six microns and stained with either 1) Delafield's haematoxylin and chromotrope, 2) Van Gieson's stain, 3) toluidine blue or Alcian blue, 4) periodic acid-Schiff's reagent (PAS) followed by Mayer's acid haemalum or 5) combinations of the preceding.

\section{RESULTS}

Observations on the living explants-Preliminary experiments indicated that synthetic medium would support the healing of fractured bones in vitro without the addition of any complex natural materials. Examination of the living explants at different times during the culture

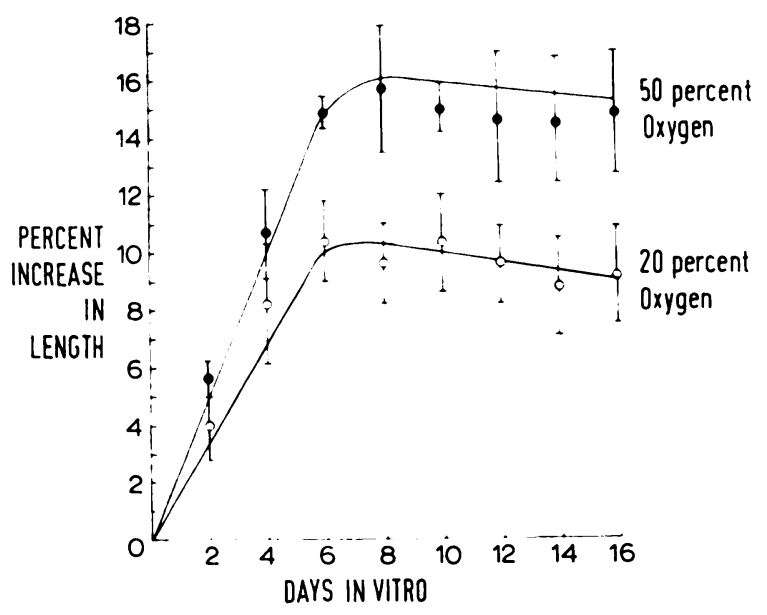

Fig. 1

Curves showing the rate of increase in length of fractured tibiae grown in 50 per cent and 20 per cent of oxygen respectively. The vertical lines indicate the standard deviations.

period led to the following general observations on the process of bone repair in vitro. During the first day in culture the cut ends of the periosteum retract and a small haemorrhage occurs at the fracture site; blood is seen to accumulate between the two bone fragments soon after the cut ends are placed together. The retracted periosteum begins to regenerate after about forty-eight hours from the time of fracture and usually the cut ends and haemorrhage are completely covered by a layer of periosteal tissue at about the fourth day; only then is it possible to turn the bone over at each change of medium. If the pieces of bone are accidentally disarranged during the first four days they seldom unite. No macroscopic changes could be seen during the later part of the culture period.

Effect of oxygen on repair-Two groups of fractured chick tibiae were grown in a gas mixture of either 20 per cent of oxygen to 75 per cent of nitrogen with 5 per cent carbon dioxide, or 50 per cent of oxygen to 45 per cent of nitrogen with 5 per cent of carbon dioxide. Figure 1 shows the increase in length of the explants at different times during the culture period. Each point on the graph is the average value of five bones, and the figure clearly shows that the higher oxygen concentration produces a significantly greater increase in the length of the 
TABLE I

The Dry Weight and Dry Weight to Wet Weight Ratio of Fractured Tibiae Grown in 50 per cent Oxygen and 20 per cent Oxygen for Eight and Sixteen Days. Also the Dry Weight and Dry Weight to Wet Weight Ratio of Intact Bones Grown in 50 per cent Oxygen for Eight and Sixteen Days. Each figure is the average of five bones, and the standard deviation is shown.

\begin{tabular}{|c|c|c|c|c|c|c|}
\hline \multirow{2}{*}{$\begin{array}{l}\text { Days } \\
\text { in vitro }\end{array}$} & \multicolumn{2}{|c|}{$\begin{array}{l}\text { Fractured bones grown in } \\
20 \text { per cent oxygen }\end{array}$} & \multicolumn{2}{|c|}{$\begin{array}{l}\text { Fractured bones grown in } \\
50 \text { per cent oxygen }\end{array}$} & \multicolumn{2}{|c|}{$\begin{array}{l}\text { Intact bones grown in } \\
50 \text { per cent oxygen }\end{array}$} \\
\hline & $\begin{array}{l}\text { Dry weight } \\
\text { (milligrams) }\end{array}$ & $\frac{\text { Dry weight }}{\text { Wet weight }} \times 100$ & $\begin{array}{c}\text { Dry weight } \\
\text { (milligrams) }\end{array}$ & $\frac{\text { Dry weight }}{\text { Wet weight }} \times 100$ & $\begin{array}{l}\text { Dry weight } \\
\text { (milligrams) }\end{array}$ & $\begin{array}{l}\text { Dry weight } \\
\text { Wet weight }\end{array} \times 100$ \\
\hline 8 & $4 \cdot 7 \pm 0.4$ & $12 \cdot 1 \pm 0 \cdot 2$ & $5 \cdot 2 \pm 0.8$ & $12 \cdot 3 \pm 0 \cdot 3$ & $4.9 \pm 0.5$ & $12 \cdot 1 \pm 0 \cdot 4$ \\
\hline 16 & $5 \cdot 2=0.4$ & $11 \cdot 3 \pm 0.4$ & $6.0 \pm 0.9$ & $12 \cdot 2=0.2$ & $5.4=0.5$ & $12.0=0.3$ \\
\hline
\end{tabular}

fractured rudiments $(0.01>p>0.001)$. The length of the explants grown in 50 per cent oxygen increases up to the eighth day and then remains constant, whereas growth in 20 per cent oxygen ceases after the sixth day. The dry weights and dry weight to wet weight ratios of fractured bones grown in the two concentrations of oxygen are shown in Table I. No significant differences in the dry weights of the fractured bones were observed, and it is clear that no significant increase in weight takes place between eight and sixteen days. The fractured bones grown for sixteen days in 20 per cent oxygen show a significant fall in dry weight to wet weight ratio, and may indicate some degenerative changes in the tissue. Table I also includes data on a set of intact bones grown under the same conditions as the fractured rudiments in 50 per cent oxygen; it will be seen that the fractured bones are not significantly different in weight from the intact bones.

Effect of different concentrations of glucoseThe effect of varying the concentration of glucose was investigated with fractured bones grown at the oxygen concentration of 50 per cent. Figure 2 shows the relation between the increase in length of the fractured bones at different times and the glucose concentration. The increase in length was the same at a concentration of either two or five milligrams of glucose per millilitre of medium, but one milligram per millilitre and eight milligrams per millilitre were both significantly less effective at the eighth and

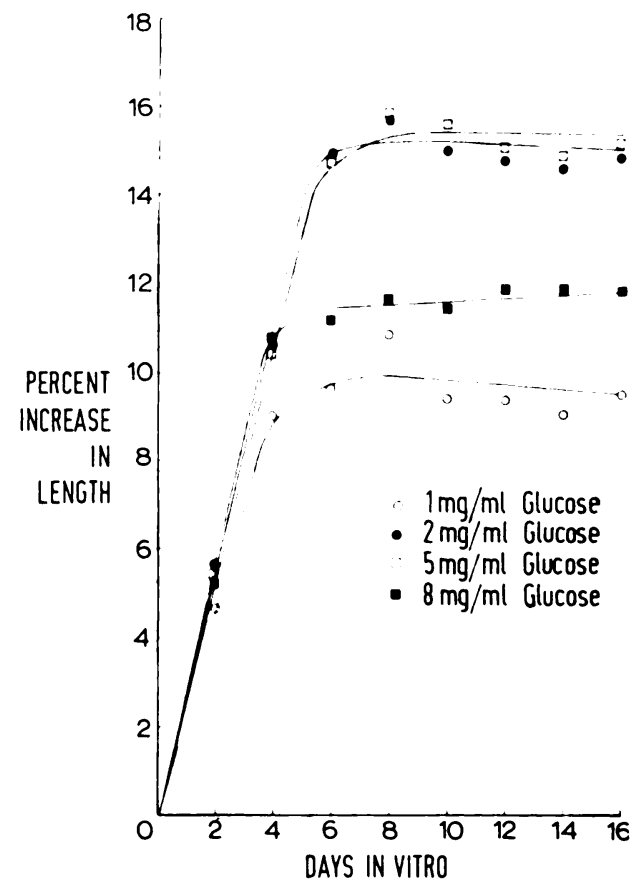

FIG. 2

Curves showing the rate of increase in length of fractured tibiae grown in medium containing varying concentrations of glucose. The best growth is obtained with two and five milligrams of glucose per millilitre.

sixteenth days. The dry weight to wet weight ratios of the fractured bones grown in different glucose concentrations are given in Table II; no significant differences were observed, except at the highest concentration of glucose, which shows a significant drop in the dry weight to wet weight ratio.

Samples of used medium were collected at the eighth and sixteenth days of culture and analysed for glucose. The relationship between the glucose concentration of the medium and the glucose metabolised by the fractured bones is illustrated in Figure 3. The amount of 
glucose removed from the medium was similar whether the medium originally contained two or five milligrams per millilitre. At a glucose concentration of one milligram per millilitre the explants were clearly becoming deficient. Above five milligrams per millilitre the amount

TABLE II

The Dry Weight to Wet Weight Ratio, the Percentage Hexosamine and Percentage Collagen of Fractured Tibiae Grown for Sixteen Days in the Presence of Varying Concentrations of Glucose Each figure is the average of four bones, and the standard deviation is shown

\begin{tabular}{|c|c|c|c|}
\hline $\begin{array}{l}\text { Concentration } \\
\text { of glucose } \\
\text { in milligrams } \\
\text { per millilitre }\end{array}$ & $\frac{\text { Dry weight }}{\text { Wet weight }} \times 100$ & $\frac{\text { Hexosamine }}{\text { Dry weight }} \times 100$ & $\frac{\text { Collagen }}{\text { Dry weight }} \times 100$ \\
\hline 1 & $12 \cdot 1 \pm 0 \cdot 6$ & $9 \cdot 1 \pm 1 \cdot 9$ & $5 \cdot 5 \pm 0.4$ \\
\hline 2 & $12 \cdot 4 \pm 0.2$ & $7 \cdot 0 \pm 0.9$ & $6 \cdot 0 \pm 0.7$ \\
\hline 5 & $12 \cdot 3 \pm 0 \cdot 3$ & $7 \cdot 4 \pm 0.6$ & $6 \cdot 2 \pm 1 \cdot 5$ \\
\hline 8 & $11 \cdot 2 \pm 0 \cdot 3$ & $7 \cdot 5 \pm 1 \cdot 2$ & $6 \cdot 8 \pm 2 \cdot 3$ \\
\hline
\end{tabular}

metabolised increased with the concentration of glucose in the medium; this increased metabolism of glucose may be connected with the degenerative changes observed in the cartilage at the highest level (see below).

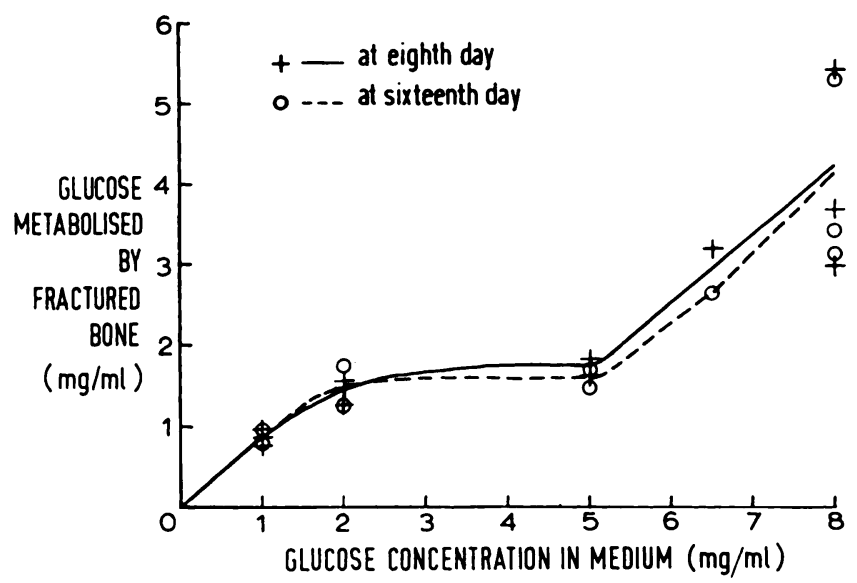

FIG. 3

Curves showing the amount of glucose utilised by the fractured tibiae in different concentrations of glucose.

Figure 4 shows the relationship between the glucose concentration of the medium and the $\mathrm{pH}$ of the used medium from the sixth to eighth day of culture. As the concentration of glucose in the medium increases, the used medium becomes more acidic.

Biochemical analysis of fractured bones-The fractured bones grown in different concentrations of glucose were analysed for total hexosamine content and collagen as measured by hydroxyproline content. All fractured bones were grown in the presence of 50 per cent oxygen. The findings are recorded in Table II. No significant differences were observed.

Histology of fractured bones-The healing of the fractured bones (two in each treatment) grown in 50 per cent oxygen was better than that of bones grown in 20 per cent oxygen, and only results obtained with the former will be described. The pattern of healing varied greatly 
according to the concentration of glucose in the medium. In the presence of one milligram of glucose per millilitre of medium there was very little cellular reaction at the fracture site (Fig. 5) and some necrosis in the shaft. In the presence of eight milligrams of glucose per millilitre fibroblasts rather than osteoblasts occupied the fracture area (Fig. 6) and some of the cells in the cartilaginous epiphyses had assumed a fibroblastic form.

The best healing was observed in medium containing two milligrams of glucose per millilitre. The repair was rather better than with five milligrams per millilitre, and vastly superior to the poor results described above with one, or with eight, milligrams per millilitre. Histological examination of fractured bones grown for four days in the presence of two milligrams of glucose per millilitre showed a completely healed periosteum and proliferating fibroblastic and osteogenic cells at the site of the break. PAS-positive material was present in this region, but there was little staining with Alcian blue or toluidine blue. On the eighth day of culture and subsequently, the fracture site was filled largely with

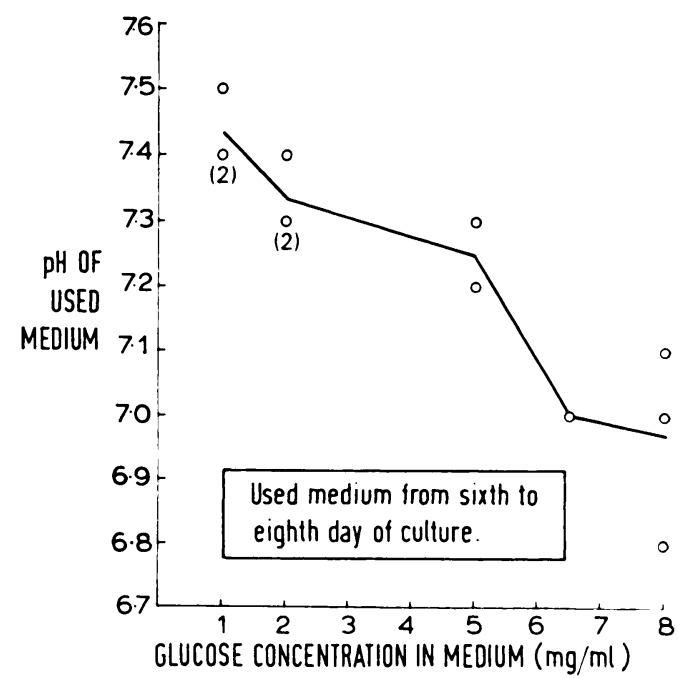

FIG. 4

Curve showing the $\mathrm{pH}$ of the used medium from the sixth to the eighth day of culture of fractured tibiae grown in different concentrations of glucose. osteogenic cells, and Van Gieson's stain indicated the presence of collagen and some new bone formation (Fig. 7). The amount of collagen increased up to the sixteenth day when the break was almost completely healed (Fig. 8). Fracture repair in excised shafts-The explants described so far had one serious drawback for the biochemical study of bone repair in vitro, in that the bone shaft was associated with large cartilaginous ends. Two further series of experiments were therefore done in which the terminal cartilage was excised. The culture conditions employed were the optimal described above, namely 50 per cent oxygen in the gas phase, and 2 milligrams of glucose per millilitre of medium. The experimental fractures were divided into two groups of six paired explants,

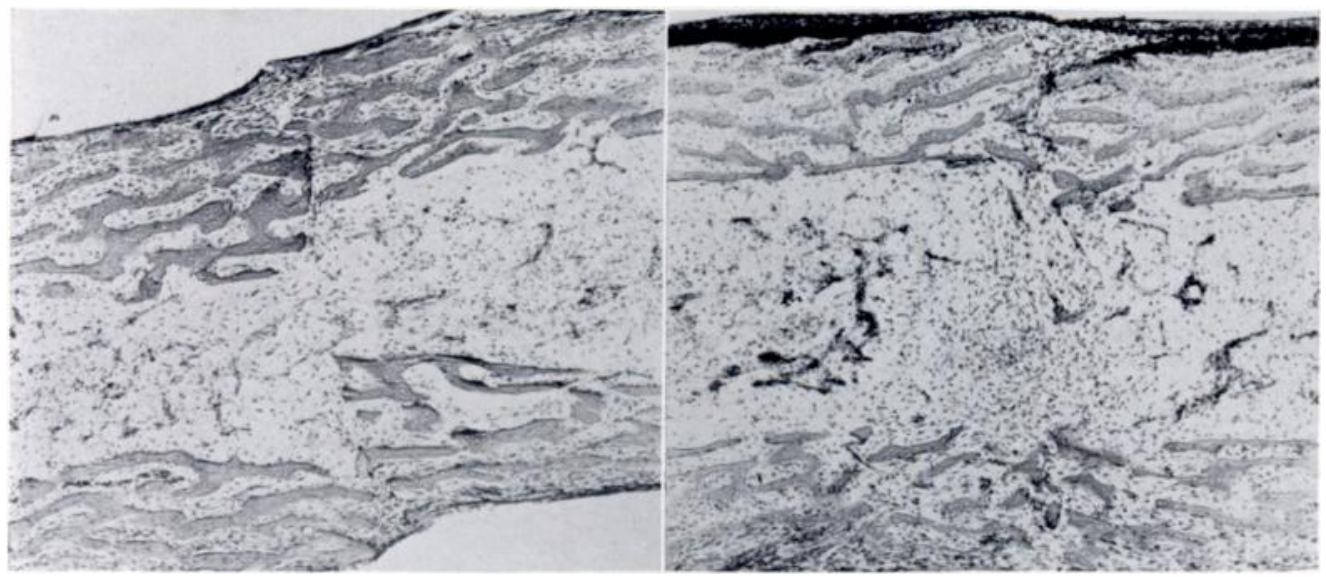

FiG. 5

FIG. 6

Figure 5-The fracture area of a tibia grown for eight days in the presence of one milligram of glucose per millilitre. Note the very slight cellular reaction at the fracture site. (Delafield's haematoxylin and chromotrope, $\times 50$.) Figure 6 - The fracture area of a tibia cultured for eight days in the presence of eight milligrams of glucose per millilitre. Note the fibroblastic reaction in the fracture area, and the absence of osteogenic cells. (Delafield's haematoxylin and chromotrope, $\times 50$.) 
one control group being cultivated in the medium just described, and the other in medium to which insulin had been added at a concentration of 500 micro-units per millilitre.

The two series of bones were examined histologically after sixteen days' growth in vitro. All six of the fractures grown in the control medium were almost completely healed (Fig. 9)

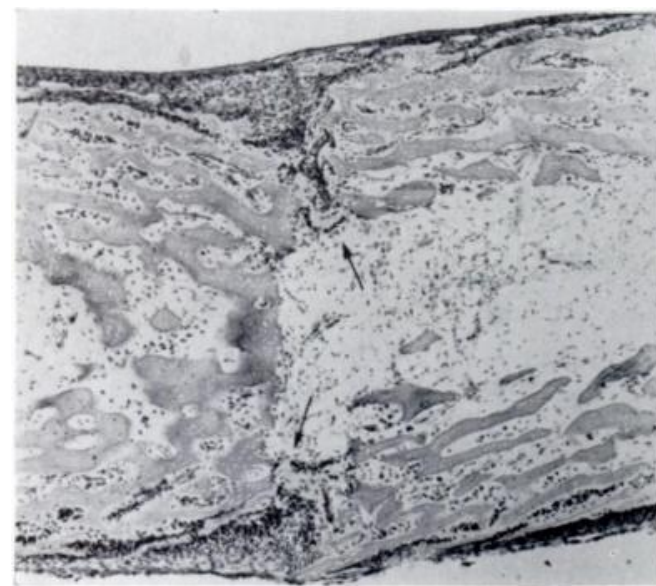

Fig. 7

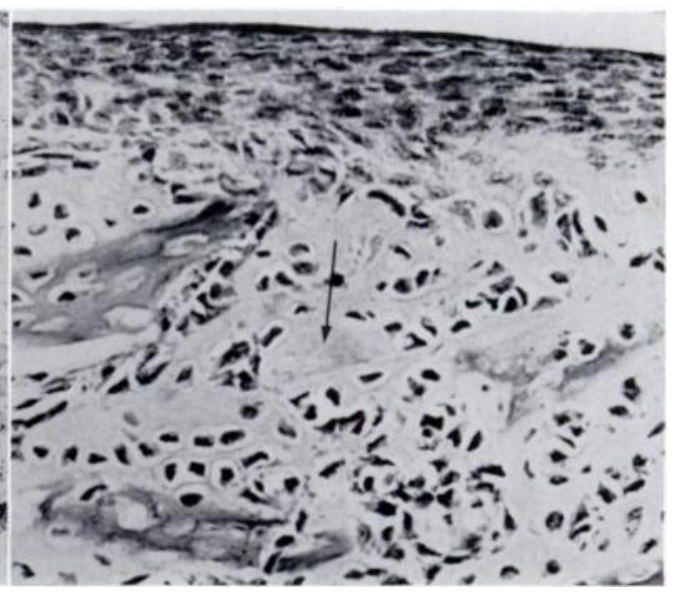

Fig. 8

Figure 7-The fracture area of a tibia grown for eight days in two milligrams of glucose per millilitre, showing the proliferation of osteogenic cells and some new bone formation bridging the gap between the two cut ends (arrowed). (Delafield's haematoxylin and chromotrope, $\times 50$.) Figure 8-Part of the fracture area of a tibia grown for sixteen days in the presence of two milligrams of glucose per millilitre, showing new bone (arrowed) filling the interstices between the original fractured trabeculae. (Delafield's haematoxylin and chromotrope, $\times 322$.)

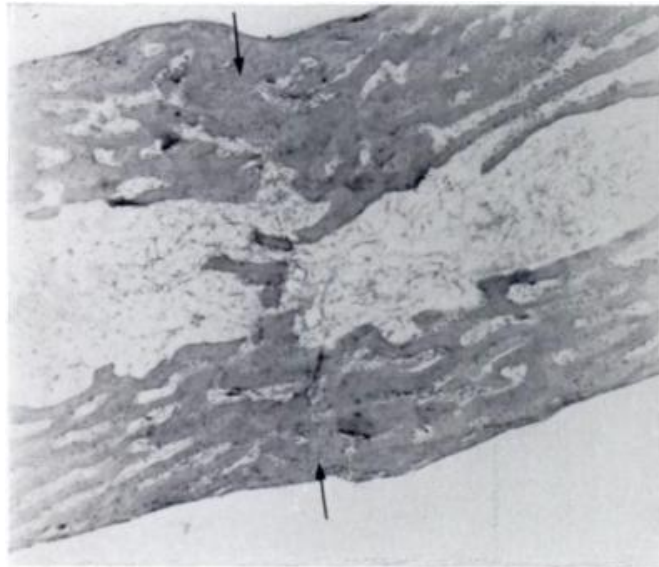

Fig. 9

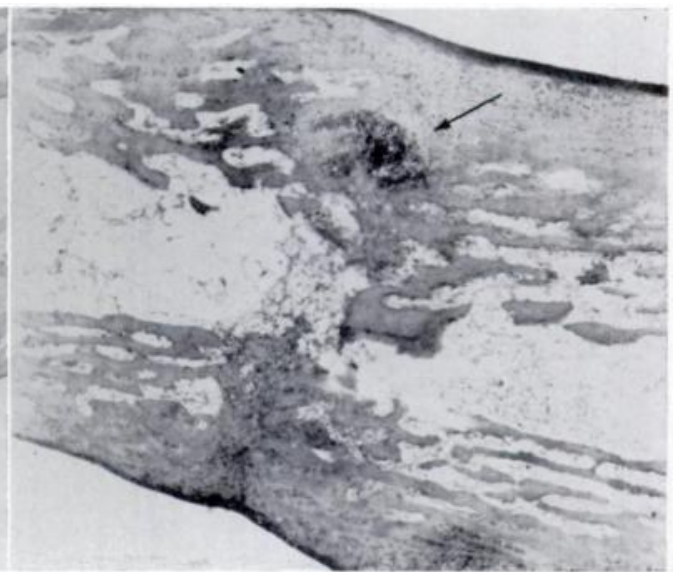

Fig. 10

Figure 9-A fractured isolated shaft grown for sixteen days in control medium. Note the complete repair of the fracture with the formation of new bone trabeculae (arrowed). (Alcian blue, periodic acid-Schiff and Van Gieson, $\times 50$.) Figure 10-A fractured isolated shaft grown in the presence of insulin for sixteen days. Note the almost complete healing and the presence of some cartilage cells in the fracture site (arrowed). (Alcian blue, periodic acid-Schiff and Van Gieson, $\times 50$.)

and new bone trabeculae had been formed; preparations stained by Van Kossa's method showed that some calcium had been deposited in these new trabeculae. In two of the fractures treated with insulin, healing was almost complete and four showed healed fracture sites which contained areas of cartilage (Fig. 10); these cartilaginous areas stained metachromatically with toluidine blue and Alcian blue. 


\section{DISCUSSION}

The results described in this paper indicate that many aspects of the repair of bone in vivo can be reproduced in vitro in bones grown in a protein-free synthetic medium. Johnson (1966) has summarised the steps in fracture healing in vivo. In the first stage there is haemorrhage at the fracture site with an extensive infiltration of cells from the damaged marrow cavity. Periosteal cells proliferate very quickly to cover the break. Similar processes take place in fractured bones in culture, except that no further contribution of cells or nutrients can be made by a blood supply. Union in vitro only occurs when the pieces of shaft are in apposition immediately after fracture, and no reaction was observed if there was any disorganisation of the fragments after two or four days in vitro. This probably indicates that the cut ends must be placed together before the blood cells that accumulate at the site of fracture degenerate.

In vivo the haemorrhage that occurs soon after fracture leads to hyperaemia in the region of the break. For this reason it was thought important to investigate the role of oxygen in the process of bone repair in vitro. The results demonstrated clearly that the rate of growth in length of fractured bones was higher in an atmosphere containing 50 per cent of oxygen than in one with 20 per cent. Histological examination showed superior healing in the higher oxygen concentration, thus emphasising the importance of an adequate oxygen supply for the proliferation of osteogenic cells after injury, and also for the process of bone repair.

The role of glucose concentration in the successful repair of fractures in vitro has been investigated in some detail, and the results showed that the most favourable concentration was two milligrams of glucose per millilitre of medium. Below this level healing was retarded and there was probably a glucose deficiency. At the highest concentration tested, eight milligrams per millilitre proliferation of osteogenic cells at the fracture site seemed to be inhibited with consequent retardation of healing. Estimations of the amount of glucose present in the used media showed that the fractured bones metabolised more glucose at the higher concentrations; this was true also of the intact bones and therefore was not a phenomenon connected with the repair process. At the highest level of glucose tested histological examination showed that some of the chondrocytes of the cartilaginous epiphyses had assumed a fibroblastic form. The $\mathrm{pH}$ of the used medium was lower at the higher concentrations of glucose possibly because of a greater conversion of glucose to lactic acid. The estimations of hexosamine in the fractured bones did not indicate any increase in polysaccharide synthesis at high levels of glucose.

Osborne and Kowalewski (1956) have shown that traumatic hyperaemia causes a much increased accumulation of sulphated mucopolysaccharides in the fracture area, and that as the healing process advances, the mucopolysaccharide content diminishes with increasing mineralisation. In vitro, it was observed that polysaccharide material was laid down in the fracture area in the early stages (PAS-positive) but that there was little staining reaction with Alcian blue or toluidine blue. However, so far, the earliest period at which an explant has been examined is four days, when the sulphated mucopolysaccharide might no longer be present. The early stages of repair in vitro obviously need a further study with particular attention to the polysaccharides in the fracture area.

The last series of experiments on excised shafts showed that the repair was similar to, if not better than, that of fractured whole bones. These isolated shafts should prove ideal for further biochemical studies. The effect of insulin is of interest, because it indicated that this hormone may be one of the factors concerned in the proliferation of cartilage cells at the fracture site in vivo; the persistence of cartilage in the regenerating area, however, delayed healing.

\section{SUMMARY}

1. The use of a protein-free synthetic medium has provided a new technical approach to the study of fracture healing in vitro.

VOL. 50 B, NO. 2, MAY 1968

$\mathbf{L}$ 
2. The tibiae of fourteen-day embryonic chicks were cut in half in the middle of the shaft, the fragments were placed in apposition and the explants grown in vitro for up to sixteen days. The process of bone repair was studied by means of histology and biochemical estimations. 3. The rate of growth in length of fractured bones was greater in an atmosphere containing 50 per cent of oxygen than in one with 20 per cent oxygen, thus emphasising the importance of an adequate oxygen supply for the regeneration of osteogenic cells.

4. The effect of varying the concentration of glucose in the medium was investigated. Two milligrams of glucose per millilitre was the most favourable for healing; higher levels caused fibroblastic changes in the cartilage cells and inhibited the proliferation of osteogenic cells at the fracture site.

5. Histological examination showed that many of the phenomena that occur in the repair of fractures in vivo can be reproduced in vitro in synthetic medium. Similar results were obtained whether the fracture was made in whole bones or in isolated shafts from which the cartilaginous ends had been removed; the latter are more favourable for biochemical study.

The authors wish to express their thanks to Professor Dame Honor B. Fell for her valuable help and encouragement. This research has been generously supported by funds from the Nuffield Foundation, and one of us (G. C. P.) was a recipient of a Commonwealth Bursary from the Royal Society and Nuffield Foundation. The figures were prepared by Mr M. Applin.

\section{REFERENCES}

Biggers, J. D., Gwatkin, R. B. L., and Heyner, S. (1961): Growth of Embryonic Avian and Mammalian Tibiae on a Relatively Simple Chemically Defined Medium. Experimental Cell Research, 25, 41.

BoAs, N. F. (1953): Method for the Determination of Hexosamine in Tissues. Journal of Biological Chemistry, 204, 553.

BUCHER, O. (1952): Untersuchungen über die Regenerationsvorgänge an experimentell gesetzten Knochenbrüchen in der Kultur in vitro. Acta Anatomica, 14, 98.

FelL, H. B. (1928): Experiments on the Differentiation in vitro of Cartilage and Bone. Part I. Archiv für experimentelle Zellforschung, 7, 390.

Fell, H. B., and Weiss, L. (1965): The Effect of Antiserum, alone and with Hydrocortisone, on Foetal Mouse Bones in Culture. Journal of Experimental Medicine, 121, 551.

Ham, A. W., and Leeson, T. S. (1961): Histology. Fourth edition. London: Pitman Medical Publishing Co. Ltd.

Johnson, L. C. (1966): The Kinetics of Skeletal Remodeling. In Structural Organization of the Skeleton, p. 66, edited by D. Bergsma. Birth Defects Original Articles Series, 1966, 2, part 1. New York: The National Foundation-March of Dimes.

McLean, F. C., and Urist, M. R. (1955): Bone, An Introduction to the Physiology of Skeletal Tissue. Chicago: The University of Chicago Press.

Niven, J. S. F. (1931): The Repair in vitro of Embryonic Skeletal Rudiments after Experimental Injury. Journal of Pathology and Bacteriology, 34, 307.

Osborne, J. C., and Kowalewski, K. (1956): The Uptake of Radiosulfur in the Fractured Humerus in the Rat. Surgery, Gynecology and Obstetrics, 103, 38.

Prockop, D. J., and Udenfriend, S. (1960): A Specific Method for the Analysis of Hydroxyproline in Tissues and Urine. Analytical Biochemistry, 1, 228.

Reynolds, J. J. (1966): The Effect of Ascorbic Acid on the Growth of Chick Bone Rudiments in Chemically Defined Medium. Experimental Cell Research, 42, 178.

Sevastikoglou, J. A. (1962): Morphological Studies of Fracture Healing in Tissue Culture. Acta Orthopaedica Scandinavica, 32, 199.

Shaw, J. L., and Bassetr, C. A. L. (1964): An Improved Method for Evaluating Osteogenesis in vitro. Anatomical Record, 149, 57.

Slein, M. W. (1963): D-Glucose, Determination with Hexokinase and Glucose-6-phosphate Dehydrogenase. In Methods of Enzymatic Analysis, p. 117. Edited by Hans-Ulrich Bergmeyer. New York and London: Academic Press.

Udupa, K. N., and Prasad, G. C. (1963): Chemical and Histochemical Studies on the Organic Constituents in Fracture Repair in Rats. Journal of Bone and Joint Surgery, 45-B, 770. 